\title{
Plasma P-Selectin Is Inversely Associated with Lung Function and Corticosteroid Responsiveness in Asthma
}

\author{
Mats W. Johansson ${ }^{a}$ Brandon M. Grilla Karina T. Barretto ${ }^{a}$ Molly C. Favour $^{a}$ \\ Hazel M. Schira ${ }^{a}$ Calvin M. Swanson ${ }^{a}$ Kristine E. Lee ${ }^{b}$ Ronald L. Sorkness ${ }^{c}$ \\ Deane F. Mosher ${ }^{a} d$ Loren C. Denlinger ${ }^{d}$ Nizar N. Jarjour ${ }^{d}$ \\ aDepartment of Biomolecular Chemistry, University of Wisconsin, Madison, WI, USA; ${ }^{b}$ Department of Biostatistics \\ and Medical Informatics, University of Wisconsin, Madison, WI, USA; 'SChool of Pharmacy, University of Wisconsin, \\ Madison, WI, USA; ${ }^{\text {dDepartment }}$ of Medicine, University of Wisconsin, Madison, WI, USA
}

\section{Keywords}

P-selectin · Asthma · Lung function · Corticosteroid ·

Phenotype $\cdot$ Exacerbation

\begin{abstract}
Background: Severe asthma has multiple phenotypes for which biomarkers are still being defined. Plasma P-selectin reports endothelial and/or platelet activation. Objective: To determine if $\mathrm{P}$-selectin is associated with features of asthma in a longitudinal study. Methods: Plasmas from 70 adult patients enrolled in the Severe Asthma Research Program (SARP) III at the University of Wisconsin-Madison were analyzed for concentration of P-selectin at several points over the course of 3 years, namely, at baseline (BPS), after intramuscular triamcinolone acetonide (TA) injection, and at 36 months after baseline. Thirty-four participants also came in during acute exacerbation and 6 weeks after exacerbation. Results: BPS correlated inversely with forced expiratory volume in $1 \mathrm{~s}\left(\mathrm{FEV}_{1}\right)$ and with residual volume/total lung capacity, an indicator of air trapping. BPS was inversely associated with $\mathrm{FEV}_{1}$ change after $\mathrm{TA}$, by regression analysis. $\mathrm{FEV}_{1}$ did not change significantly after TA if BPS was above the median, whereas patients with BPS below the median had sig-
\end{abstract}

nificantly increased $\mathrm{FEV}_{1}$ after TA. BPS was higher in and predicted assignment to SARP phenotype cluster 5 ("severe fixed-airflow asthma"). P-selectin was modestly but significantly increased at exacerbation but returned to baseline within 3 years. Conclusions: High BPS is associated with airway obstruction, air trapping, the "severe fixed-airflow" cluster, and lack of $\mathrm{FEV}_{1}$ improvement in response to TA injection. P-selectin concentration, which is a stable trait with only modest elevation during exacerbation, may be a useful biomarker for a severe asthma pheno- or endotype characterized by low pulmonary function and lack of corticosteroid responsiveness.

๑) 2020 S. Karger AG, Basel

\section{Introduction}

Severe asthma has multiple phenotypes for which biomarkers are still being defined [1-13]. One potential biomarker is plasma P-selectin [14-21]. P-selectin is a type I membrane protein that is sequestered in Weibel-Palade

Edited by: H.-U. Simon, Bern.

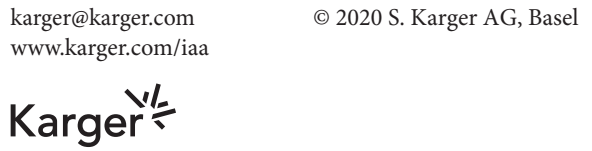

Mats W. Johansson

Department of Biomolecular Chemistry, University of Wisconsin School of Medicine and Public Health, 1135 Hector F. DeLuca Biochemistry Building, 420 Henry Mall, Madison, WI 53706 (USA)

mwjohansson@wisc.edu 
Table 1. Patient characteristics at baseline

Subjects, $N$

Asthma severity: severe, $n(\%)$; nonsevere, $n(\%)$

Sex: female, $n$ (\%); male, $n$ (\%)

Age, years, median (quartiles)

Race/ethnicity: white non-Hispanic; black; Asian American; Hispanic, $n$ (\%) for each group

Pre-BD FEV $1, \%$ predicted, mean \pm SD

Median (quartiles)

Pre-BD FEV 1 /FVC, \% predicted, median (quartiles)

Pre-BD FVC, $\%$ predicted, mean \pm SD

Pre-BD RV/TLC, \% predicted, mean \pm SD

Post-BD FEV 1 , \% predicted, mean $\pm \mathrm{SD}$

Median (quartiles)

Post-BD change in $\mathrm{FEV}_{1}, \%$ predicted, median (quartiles)

On ICS, $n(\%)$

On OCS, $n(\%)$

ICS dose, $\mu \mathrm{g} /$ day, mean \pm SD

\section{0}

$46(66) ; 24(34)$

$45(64) ; 25(36)$

$48(33,58)$

$60(86) ; 9(13) ; 1(1) ; 3$ (4)

$78 \pm 20$

$78(62,92)$

$91(84,96)$

$86 \pm 17$

$116 \pm 18$

$86 \pm 20$

$84(73,102)$

$8(5,11)$

$60(86)$

$6(9)$

$740 \pm 420$

Data are baseline data and presented as mean \pm SD (if data are normally distributed) or median (25th, 75th percentiles) (if data are not normally distributed). BD, bronchodilation; $\mathrm{FEV}_{1}$, forced expiratory volume in $1 \mathrm{~s}$; FVC, forced vital capacity; ICS, inhaled corticosteroid; OCS, oral corticosteroid; RV, residual volume; TLC, total lung capacity.

granules of endothelial cells and $\alpha$-granules of platelets and is mobilized in response to various inflammatory and thrombogenic mediators, resulting in cell-surface display and subsequent proteolytic release into blood plasma [14-18, 22-26]. It is found soluble in plasma in normal subjects, with reported median values ranging from about 10 to $40 \mathrm{ng} / \mathrm{mL}$ or more, depending on anticoagulant, plasma preparation protocol, and detection platform [14, 27-29]. Plasma P-selectin is elevated under certain circumstances in asthma [14, 16-19]. It has been reported to be increased up to 3 -fold in other diseases, including hemolytic-uremic syndrome, thrombotic thrombocytopenic purpura, acute myocardial infarction, aspirin-exacerbated respiratory disease, aspirin-intolerant urticaria, chronic obstructive pulmonary disease, and in human immunodeficiency disease, and to be associated with features of interstitial lung disease [14, 20-22, 27, 30-32].

In the Severe Asthma Research Program (SARP), phase II, which was an observational study in which the cohort was classified in 5 asthma phenotypes using unsupervised hierarchical cluster analysis [33], plasma P-selectin analyzed in University of Wisconsin-Madison patients was higher in a pool of the 3 more severe phenotype clusters [16]. Furthermore, higher plasma P-selectin levels correlated with greater area of low density on chest computed tomography (CT) at total lung capacity (TLC), whereas the platelet-specific marker platelet factor $4 \mathrm{did}$ not correlate with the CT signal, indicating that P-selectin in asthma likely mainly reports endothelial activation [16]. Following whole-lung allergen challenge in (nonSARP) patients with mild allergic asthma, a human model of asthma exacerbation [34] known to cause platelet activation [17], there was a transient modest increase in $\mathrm{P}$-selectin [16], but the variation in P-selectin among time points was less than the variation among subjects [16]. Overall, these findings indicate that asthma patients have variable levels of plasma $\mathrm{P}$-selectin that correlate with disease features and are perturbed only transiently by exacerbation.

The relationships of plasma $\mathrm{P}$-selectin with asthma clusters and airway structural change described above were determined at single points in time, and the wholelung challenge study lacks the complexity of a natural exacerbation $[15,16]$. Associations between aspects of asthma and the stability of P-selectin levels over time, including exacerbations in asthma, have not been examined. We therefore undertook the present investigation to address these unknowns in Wisconsin patients participating in the longitudinal SARP III, which included an intramuscular triamcinolone acetonide (TA) injection and visits during and after exacerbations. 


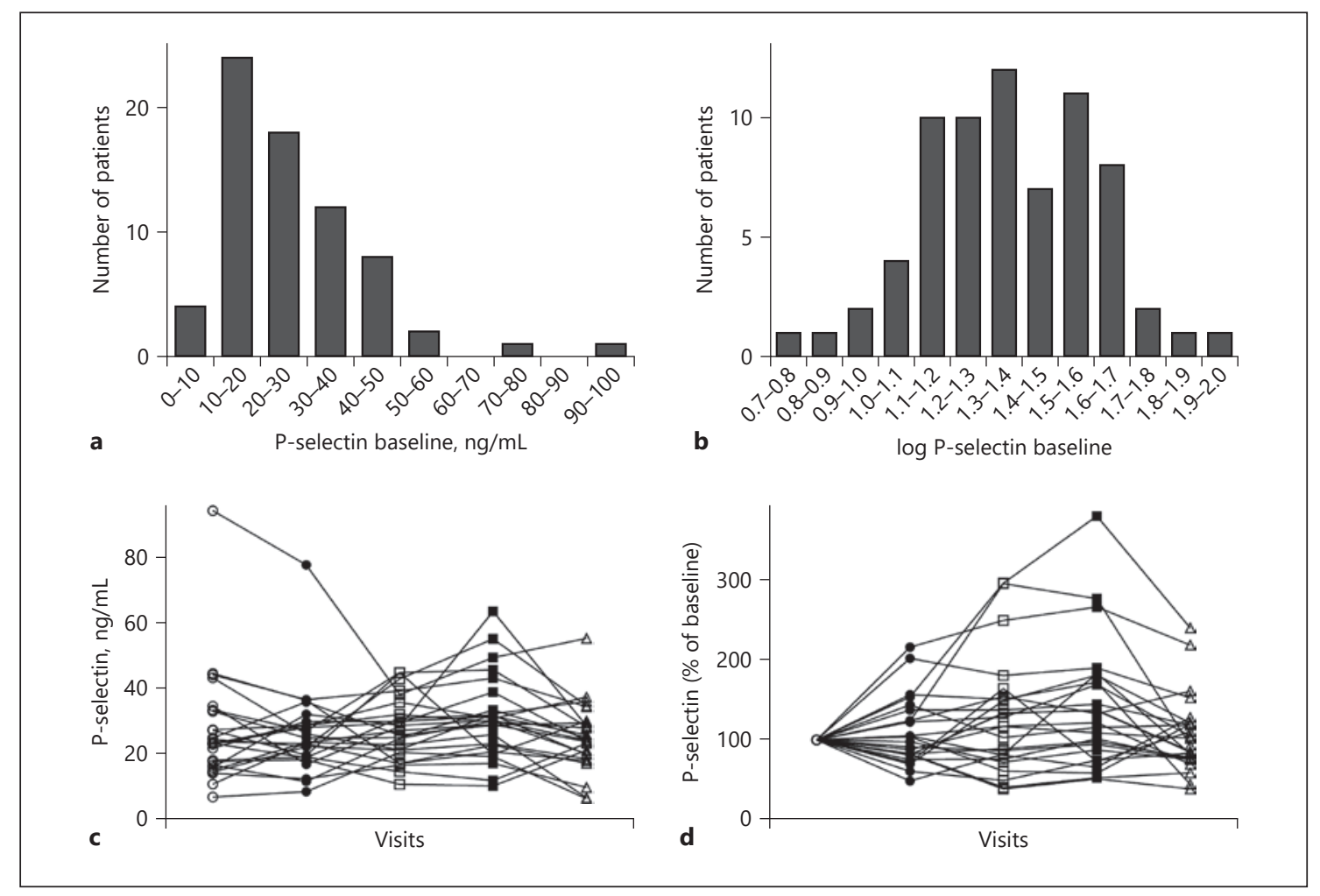

Fig. 1. a Distribution of BPS in patients grouped by decades of concentration in $\mathrm{ng} / \mathrm{mL}$. $\mathbf{b}$ Distribution of BPS in patients grouped by log concentration. c Plasma P-selectin concentration over time in the 25 patients with an asthma exacerbation, from whom samples were available from all 5 visits. Symbols for visits: open circle, baseline; filled circle, after TA administration; open square, acute exacerbation; filled square, recovery after exacerbation; open triangle, final visit 36 months after baseline. d Plasma P-selectin as percentage of baseline in the same patients as in c. BPS, baseline P-selectin; TA, triamcinolone acetonide.

\section{Materials and Methods}

\section{Patients}

Patients were recruited at the University of Wisconsin-Madison to participate in the National Heart, Lung, and Blood Institute's SARP III [35-37], which was a Health Insurance Portability and Accountability Act (HIPAA)-compliant study. Participants included adult patients with nonsevere or severe asthma (Table 1), according to a modification of the European Respiratory Society/ American Thoracic Society definition [35]. The study subjects are a well-characterized population [35-39].

\section{Study Visits and Assessments}

Patients were characterized at baseline $[35,36]$. Patients received a single dose of 40-mg intramuscular triamcinolone acetonide (TA) given deep in the gluteal region as described [36]. The response was evaluated at a post-TA visit $18 \pm 3$ days after TA administration [36]. Following the baseline and TA-response characterizations, some participants also came in during a subsequent exacerbation. The acute exacerbation visit was within 5 days of a subject reporting $\geq 2$ days of increased symptoms compared with baseline and could occur any time after the subject had completed the baseline and post-TA visits and at least 6 weeks prior to the final visit [37], which was 36 months after baseline [38]. The recovery visit was 6 weeks after the acute exacerbation visit. Assessments were performed as before and described [15, 16, 36, 40, 41].

\section{Enzyme-Linked Immunosorbent Assay}

Plasma was collected by a procedure that minimizes artifactual platelet activation as before $[15,16]$. In brief, blood was collected in vacuum tubes containing CTAD (citrate, theophylline, adenosine, and dipyridamole) anticoagulant solution (BD Vacutainer Systems, Franklin Lakes, NJ, USA), the sample was double-spun, and the final supernatant was stored in aliquots at $-80^{\circ} \mathrm{C}$ until tested [15]. The concentration of soluble P-selectin was determined using sandwich enzyme-linked immunosorbent assay (ELISA) (R\&D Systems, Minneapolis, MN, USA), according to the manufacturer's instructions, as before [15], except that samples were diluted $1: 20,1: 30$, and 1:40. The values within the range of the standards were averaged [15]. The absorbance of the colored product was measured at $450 \mathrm{~nm}$, with wavelength correction at 620 $\mathrm{nm}$, in a SpectraMax M5 plate reader (Molecular Devices, Sunnyvale, CA, USA). Each dilution was run in duplicate [15]. The detection limit was $5 \mathrm{ng} / \mathrm{mL}$ [15]. Values were not adjusted for the dilution by the anticoagulant solution present in the tube, for the reason given before [15]. An aliquot of pooled plasma collected from 
Table 2. Correlations between BPS concentration and $\mathrm{FEV}_{1}(\%$ predicted) or RV/TLC (\% predicted) at baseline

\begin{tabular}{llllll}
\hline Group $(n)$ & \multicolumn{2}{c}{$\mathrm{FEV}_{1}, \%$ predicted } & & \multicolumn{2}{l}{ RV/TLC, \% predicted } \\
\cline { 2 - 3 } & $r_{\mathrm{s}}$ & $p$ value & & $r_{\mathrm{s}}$ & $p$ value \\
\hline All (70) & -0.30 & 0.01 & & 0.28 & 0.03 \\
Severe asthma (46) & -0.34 & 0.02 & & 0.25 & 0.12 \\
Nonsevere asthma (24) & -0.20 & 0.34 & & 0.31 & 0.17 \\
\hline
\end{tabular}

$\log$ P-selectin correlated with $\mathrm{FEV}_{1}$ or RV/TLC (which were normally distributed) with Pearson correlation coefficient $(r)=$ $-0.30, p=0.01$ or $r=0.30, p=0.02$ in all patients, respectively. $\mathrm{FEV}_{1}$, prebronchodilation $\mathrm{FEV}_{1}$; BPS, baseline P-selectin; $\mathrm{FEV}_{1}$, forced expiratory volume in $1 \mathrm{~s} ; r_{\mathrm{s}}$, Spearman rank correlation coefficient; RV, residual volume; TLC, total lung capacity.

8 subjects and handled the same as test plasma was analyzed alongside the test sample to ensure the stability of the ELISA over time. The concentration of $\mathrm{P}$-selectin in the pooled sample measured on 26 occasions was $30.7 \pm 1.7 \mathrm{ng} / \mathrm{mL}$ (mean $\pm \mathrm{SE}$ of the mean).

\section{Statistical Analysis}

The paired $t$ test or Wilcoxon matched-pair signed-rank test was used to compare data between visits, if the variable was normally distributed (passed Prism's normality test) or was not normally distributed, respectively. The unpaired $t$ test or Mann-Whitney $U$ test was used to compare ordinal data between 2 groups, if the variable was or was not normally distributed. One-way analysis of variance (ANOVA) or the Kruskal-Wallis test was used to compare ordinal data among groups, if the variable was or was not normally distributed. Fisher's exact probability test was used in contingency table analysis to compare nominal data between 2 groups. The Spearman rank test was used to analyze correlations. In some cases, the Pearson test was used to analyze correlations between normally distributed or log-transformed variables. Unadjusted or adjusted linear regression models were used to analyze the effects of P-selectin as the independent predictor variable on dependent outcome variables. A level of probability $(p) \leq 0.05$ was considered significant. Analyses were performed using Prism (GraphPad, San Diego, CA, USA) or SAS (Cary, NC, USA). Group data are reported as mean \pm SD if the variable was normally distributed and as median with 25 th and 75 th percentiles if the variable was not normally distributed.

\section{Results}

\section{Patients}

ELISA for soluble plasma $\mathrm{P}$-selectin was performed on samples from 70 University of Wisconsin-Madison SARP III patients, of whom $66 \%$ had severe asthma and $34 \%$ had nonsevere asthma (Table 1). Other patient characteristics at baseline are also described in Table 1. Criteria for severe and nonsevere asthma were previously described [35].
Baseline P-Selectin Correlates with FEV $\mathrm{F}_{1}$ or RV/TLC

The coefficient of variation $(\mathrm{CV})$ for plasma P-selectin concentration among subjects at baseline was 59\%, that is, there was considerable patient variability (Fig. 1a, b). Two patients had a very high baseline P-selectin (BPS) of $>70$ $\mathrm{ng} / \mathrm{mL}$ that decreased in subsequent samples (Fig. 1c and not shown). BPS was not normally distributed (Fig. 1a), but log transformation resulted in a normal distribution (Fig. 1b). BPS did not correlate with age (Spearman rank correlation coefficient $\left.\left[r_{\mathrm{s}}\right]=-0.00 ; p=0.97\right)$. Also, BPS was not significantly different between the sexes (median [quartiles] in females $=21.8 \mathrm{ng} / \mathrm{mL}[14.5,34.0]$ and in males $=25.9 \mathrm{ng} / \mathrm{mL}[16.6,34.5] ; p=0.37)$. BPS correlated inversely with baseline $\mathrm{FEV}_{1}$ (prebronchodilation [BD], as percentage of the predicted value) in all patients or in patients with severe asthma (Table 2) and was associated with baseline (pre-BD) $\mathrm{FEV}_{1}$ by regression analysis, with somewhat lower $p$ value after adjustment for age and severity (Table 3 ). Similarly, BPS also correlated inversely with baseline post-BD FEV ${ }_{1}\left(r_{\mathrm{s}}=-0.25, p=0.04\right)$, including patients with severe asthma $\left(r_{s}=-0.29, p=0.05\right)$. Air trapping, like decreased $\mathrm{FEV}_{1}$, is a feature of severe asthma [40]. BPS correlated and was associated with percentage of predicted residual volume as fraction of total lung capacity (RV/TLC), an indicator of air trapping, regardless whether adjusting for age and severity [40] (Tables 2, 3). However, BPS was no longer associated with RV/TLC after additional adjustment for $\mathrm{FEV}_{1}$ (Table 3 ), presumably because $\mathrm{FEV}_{1}$ and RV/TLC correlate with each other $\left(r_{\mathrm{s}}=\right.$ $-0.79, p<0.001)$. Taken together, these data indicate that high BPS is associated with airway obstruction or decreased pulmonary function, as reported by $\mathrm{FEV}_{1}$, and with air trapping, as reported by RV/TLC.

\section{$B P S$ Is Inversely Associated with FEV $V_{1}$ Change in Response to TA}

The baseline evaluation included an injection with TA, after which the participants returned for repeat assessments $18 \pm 3$ days later. After the TA administration, there was a trend to lower $\mathrm{P}$-selectin compared with baseline (Table 4; Fig. 1c, d) $(p=0.12)$. BPS correlated with change in P-selectin after TA in the current study $\left(r_{\mathrm{s}}=\right.$ $-0.74, p<0.001$ ), that is, the largest decreases in P-selectin in response to TA occurred in patients with highest BPS. In consistency with the network-wide SARP III population, in which the TA injection led to variable changes among subjects in $\mathrm{FEV}_{1}$ [36], variable changes in $\mathrm{FEV}_{1}$ were observed in the present subjects. BPS was inversely associated with (pre-BD) $\mathrm{FEV}_{1}$ change after TA by regression analysis, after adjustment for age, severity, and 
Table 3. Linear regression models for the effect of BPS on $\mathrm{FEV}_{1}$ (\% predicted), RV/TLC (\% predicted) at baseline, or FEV $\mathrm{change}$ (\% predicted) after intramuscular TA administration

\begin{tabular}{|c|c|c|c|c|c|c|}
\hline \multirow[t]{2}{*}{ Model } & \multicolumn{2}{|l|}{$\mathrm{FEV}_{1}, \%$ predicted } & \multicolumn{2}{|c|}{ RV/TLC, \% predicted } & \multicolumn{2}{|c|}{$\mathrm{FEV}_{1}, \%$ predicted change after TA } \\
\hline & $\beta(95 \% \mathrm{CI})$ & $p$ value & $\beta(95 \% \mathrm{CI})$ & $p$ value & $\beta(95 \% \mathrm{CI})$ & $p$ value \\
\hline Unadjusted & $-6.1(-10.4,-1.8)$ & 0.007 & $6.0(2.2,9.9)$ & 0.003 & $-1.4(-3.5,0.6)$ & 0.17 \\
\hline Adjusted for age and severity & $-6.1(-9.9,-2.2)$ & 0.003 & $5.7(1.9,9.5)$ & 0.004 & $-1.8(-3.8,0.2)$ & 0.09 \\
\hline Adjusted for age, severity, $\mathrm{FEV}_{1}$ & - & & $1.2(-1.5,3.8)$ & 0.40 & $-2.6(-4.6,-0.4)$ & 0.02 \\
\hline
\end{tabular}

$\beta$ estimate for the association with BPS, showing the amount of difference in a lung function variable or in the $\mathrm{FEV}_{1}$ change variable

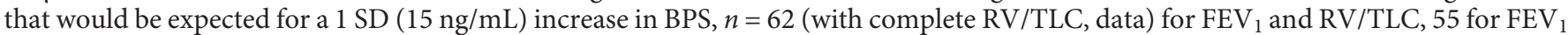
change. TA, triamcinolone acetonide; $\mathrm{FEV}_{1}$, prebronchodilation $\mathrm{FEV}_{1}$. With $n=70, \beta$ was $-6.9(-11.3,-2.5), p=0.002$, for FEV $\mathrm{FPS}_{\text {, }}$ baseline P-selectin; CI, confidence interval; $\mathrm{FEV}_{1}$, forced expiratory volume in $1 \mathrm{~s} ; r_{\mathrm{s}}$, Spearman rank correlation coefficient; RV, residual volume; TLC, total lung capacity.

Table 4. Plasma P-selectin concentration at baseline, after intramuscular TA administration, during exacerbation and recovery, and 36 months after baseline

\begin{tabular}{|c|c|c|c|c|c|}
\hline \multirow[t]{2}{*}{ Variable } & \multicolumn{5}{|l|}{ Visit } \\
\hline & baseline & post-TA & $\begin{array}{l}\text { acute } \\
\text { exacerbation }\end{array}$ & $\begin{array}{l}\text { recovery after } \\
\text { exacerbation }\end{array}$ & $\begin{array}{l}36 \text { months after } \\
\text { baseline }\end{array}$ \\
\hline P-selectin, ng/mL & $23.2(15.6,34.4)$ & $22.4(16.9,29.4)$ & $26.4(19.8,33.0)$ & $30.0(21.3,34.8)^{\mathrm{a}, \mathrm{b}}$ & $23.2(18.2,29.4)$ \\
\hline P-selectin, $\%$ of baseline & 100 & $92(66,139)$ & $117(79,152)^{\mathrm{a}}$ & $127(84,174)^{\mathrm{a}, \mathrm{c}-\mathrm{e}}$ & $89(75,122)$ \\
\hline
\end{tabular}

Data are presented as median (25th, 75th percentiles); $n=70,70,26,26$, and 60 for the different visits, respectively. TA, triamcinolone acetonide. ${ }^{\mathrm{a}} p \leq 0.05$ versus the 36 -month visit. ${ }^{\mathrm{b}} p \leq 0.01$ versus the acute exacerbation visit. ${ }^{\mathrm{c}} p \leq 0.05$ versus the acute exacerbation visit. ${ }^{\mathrm{d}} p \leq 0.05$ versus the baseline visit. ${ }^{\mathrm{e}} p \leq 0.05$ versus the post-TA visit.

baseline $\mathrm{FEV}_{1}$ (Table 3). When dividing the patients according to median BPS, BPS-high patients did not have a significant increase in $\mathrm{FEV}_{1}$ after TA compared with baseline, whereas BPS-low patients had significantly higher $\mathrm{FEV}_{1}$ after TA (Table 5). Dividing patients by BPS quartiles, the 1st quartile (with the lowest BPS) had significantly higher $\mathrm{FEV}_{1}$ after TA and the other quartiles had no significant change in $\mathrm{FEV}_{1}$ after TA (online suppl. Table 1; see www.karger.com/doi/10.1159/000509600 for all online suppl. material). Furthermore, there was a significant difference in $\mathrm{FEV}_{1}$ among the quartiles at baseline $(p=0.006$, ANOVA $)$ and after TA $(p=0.002)$, with the 4th quartile (with the highest BPS) having significantly lower $\mathrm{FEV}_{1}$ than the other quartiles at both visits (online suppl. Table 1). BPS did not correlate significantly with post-BD change in $\mathrm{FEV}_{1}$ (i.e., change from baseline pre-BD FEV $\mathrm{F}_{1}$ to baseline post-BD $\mathrm{FEV}_{1} ; r_{\mathrm{s}}=0.01, p=$ $0.91)$. Overall, these data indicate that high BPS is associated with lack of response to injected steroid, whereas low BPS is associated with significant improvement in $\mathrm{FEV}_{1}$ after injected steroid.

P-Selectin Is Inversely Associated with Corticosteroid Responsiveness in Asthma
Table 5. $\mathrm{FEV}_{1}$ (\% predicted) at baseline and after intramuscular TA administration in BPS-low (below median BPS) and BPS-high (above median) patients

\begin{tabular}{llll}
\hline Group $(n)$ & \multicolumn{2}{l}{ Visit } & \\
\cline { 2 - 4 } & baseline & post-TA & $p$ value \\
\hline Below median/BPS-low (27) & $84 \pm 19$ & $87 \pm 20$ & 0.002 \\
Above median/BPS-high (27) & $74 \pm 22$ & $75 \pm 22^{\text {a }}$ & 0.43 \\
\hline
\end{tabular}

Data are presented as mean \pm SD. TA, triamcinolone acetonide; $\mathrm{FEV}_{1}$, prebronchodilation $\mathrm{FEV}_{1}$; BPS, baseline P-selectin; $\mathrm{FEV}_{1}$, forced expiratory volume in $1 \mathrm{~s}$. ${ }^{\mathrm{a}} p \leq 0.05$ versus BPS-low $(<23.1$ $\mathrm{ng} / \mathrm{mL}$ ).

BPS Is Not Associated with Type 2 Immunity, Is Higher in Asthma Phenotype Cluster 5, and Predicts Assignment to This Cluster

Asthma can be analyzed and categorized in different ways, including severity, degree of type 2 immunity, and by cluster analysis. We therefore placed our subjects in 
Table 6. Characteristics of BPS-high and BPS-low patients at baseline

\begin{tabular}{lccc}
\hline Variable & BPS-high & BPS-low & $p$ value \\
\hline Age, years, median (quartiles) & $48(32,56)$ & $48(35,62)$ & 0.56 \\
Sex: female, $n$ (\%); male, $n$ (\%) & $20(57) ; 15(43)$ & $25(71) ; 10(29)$ & 0.32 \\
Serum IgE, IU/mL, median (quartiles) & $80(30,250)$ & $120(40,200)$ & 0.74 \\
Blood eosinophils, \%, median (quartiles) & $4(2,5)$ & $4(2,6)$ & 0.68 \\
Blood eosinophils, per $\mu \mathrm{L}$, median (quartiles) & $250(130,370)$ & $220(150,400)$ & 0.95 \\
Blood neutrophils, \%, median (quartiles) & $56(52,62)$ & $60(47,64)$ & 0.91 \\
Blood neutrophils, per $\mu \mathrm{L}$, median (quartiles) & $3,700(3,000,4,900)$ & $3,800(2,600,4,400)$ & 0.58 \\
Sputum eosinophils, \%, median (quartiles) & $0.2(0.0,1.2)$ & $0.9(0.0,3.9)$ & 0.20 \\
Sputum neutrophils, \%, mean \pm SD & $61 \pm 22$ & $66 \pm 21$ & 0.40 \\
FENO, ppb, median (quartiles) & $22(12,36)$ & $20(12,34)$ & 0.77 \\
\hline
\end{tabular}

Data are presented as mean \pm SD (if data are normally distributed) or median (25th and 75th percentiles) (if data are not normally distributed). BPS-high $\geq$ median, BPS-low $<$ median. BPS, baseline P-selectin; FENO, fraction of exhaled nitric oxide; Ig, immunoglobulin.

Fig. 2. a BPS in patients assigned to different asthma phenotype clusters. Using the 5 phenotype groups identified in SARP II [1], present SARP III subjects have been assigned at baseline to the 5 clusters using a 11-variable model (data provided by the SARP III DCC). Cluster 1 = "mild allergic asthma," 2 = "mild-moderate allergic asthma," 3 = "more severe older-onset asthma," 4 = "severe variable allergic asthma," and 5 = "severe fixed-airflow asthma" [1]; $N=$ $70(10+29+9+8+14)$. Bar $=$ median; ** $p<0.01$ versus cluster 1 or 2 ; ${ }^{*} p<0.05$ versus cluster 3 or 4 . $\mathbf{b}$ ROC curve for the ability of BPS to predict assignment to cluster 5. Area under curve $=0.77(p=0.002)$; for the statistically optimal criterion of $>34.5 \mathrm{ng} / \mathrm{mL}$, specificity $=86 \%$ and sensitivity $=57 \%$. BPS, baseline P-selectin; DCC, Data Coordinating Center; ROC, receiver operating characteristic.

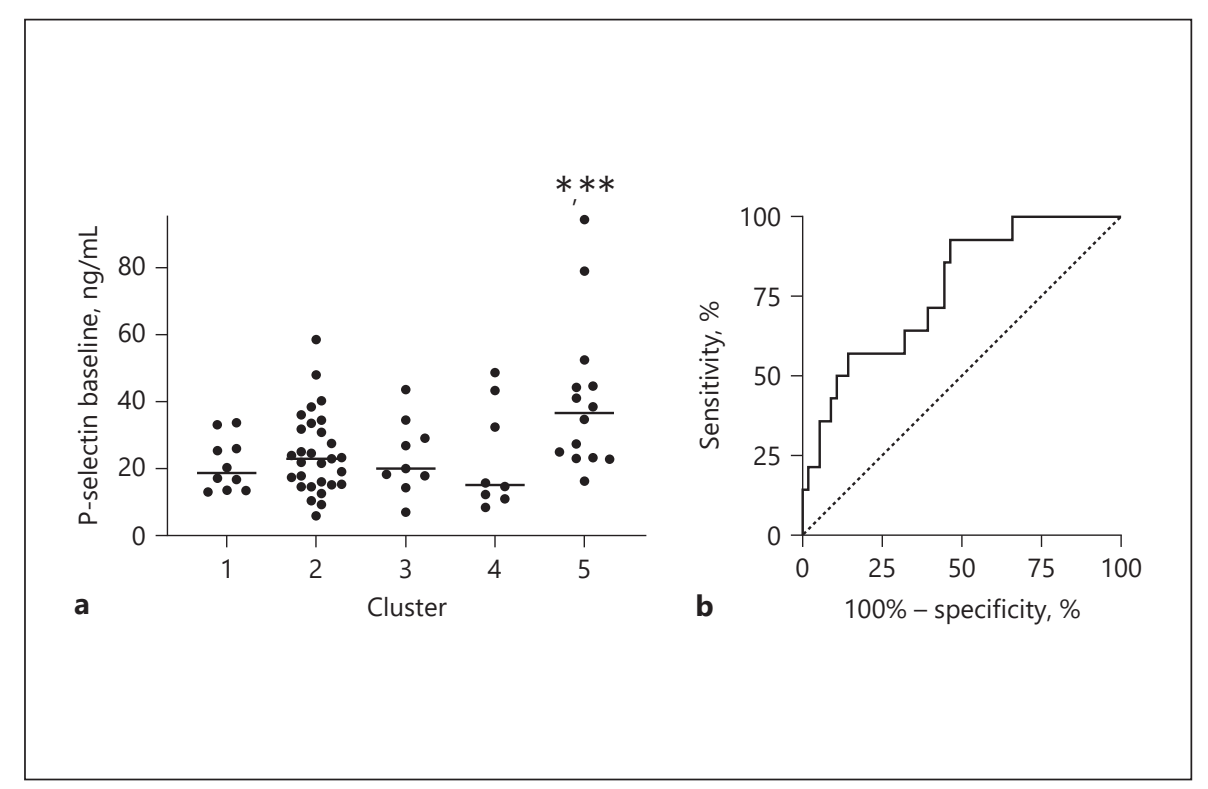

such subcategories including investigating whether BPS is associated with type 2 immunity inflammation, which is present in many patients with asthma [1-3, 5-13]. Fraction of exhaled nitric oxide (FENO) or sputum eosinophils are associated with corticosteroid responsiveness [36] and blood eosinophils are associated with reported exacerbations in the past year [37] in the general SARP III population. BPS did not correlate significantly with any of the type 2 immunity variables, blood and sputum eosinophils, FENO, or serum immunoglobulin (Ig) $E\left(r_{\mathrm{s}}=\right.$ $0.00, p=0.98$ for blood eosinophil percentage; $r_{\mathrm{s}}=0.02$, $p=0.86$ for blood eosinophil concentration; $r_{\mathrm{s}}=-0.11$, $p=0.39$ for sputum eosinophil percentage; $r_{\mathrm{s}}=0.04, p=$ 0.75 for FENO; and $r_{\mathrm{s}}=0.01, p=0.92$ for IgE). To characterize further the patients with high BPS, values for the type 2 immunity variables as well as for blood and sputum neutrophils in the BPS-high group (divided by median as shown above) compared with the BPS-low group are shown in Table 6. BPS-high patients were not significantly different from the BPS-low ones with respect to these variables (Table 6). Baseline FENO and sputum eosinophils correlated positively with (pre-BD) $\mathrm{FEV}_{1}$ change af- 
ter TA $\left(r_{\mathrm{s}}=0.44\right.$ and $0.39 ; p=0.001$ and 0.006 , respectively), consistent with associations in the general SARP III cohort [36]. In the current population, blood eosinophil percentage and concentration and IgE also correlated with $\mathrm{FEV}_{1}$ change after TA $\left(r_{\mathrm{s}}=0.30,0.28\right.$, and 0.34 ; $p=0.03,0.04$, and 0.01 , respectively). In the general SARP

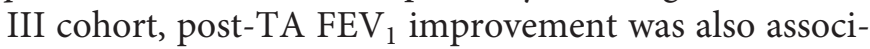
ated with baseline BD response [36].

To investigate whether there was any relationship between plasma P-selectin and the 5 asthma phenotype clusters identified in SARP II [33], we examined BPS levels in the current Wisconsin SARP III patients, who have been assigned at baseline to the 5 clusters using a 11-variable model (data provided by the SARP III Data Coordinating Center [DCC]). There was a significant difference in BPS concentration among the 5 clusters $(p=0.03$, Kruskal-Wallis test), such that BPS was higher in cluster 5 (called "severe fixed-airflow asthma" [33]) than in the other clusters (Fig. 2a). Receiver operating characteristic (ROC) curve analysis demonstrated that BPS significantly predicted assignment to cluster 5 (Fig. 2b), indicating that BPS is a potential biomarker for cluster 5 assignment.

\section{P-Selectin Is Modestly Increased at a Natural Asthma}

Exacerbation but Otherwise Is Stable over Time

Of the 70 subjects, 26 (37\%) reported at least 1 exacerbation during the 3 years of the study and came in for an acute exacerbation visit and 6 weeks later for a recovery visit (Table 4; Fig. 1c, d). P-selectin was modestly but significantly increased at the acute exacerbation visit and remained significantly elevated at the recovery visit but not at the 36 months after baseline visit. At the 36-month visit, P-selectin was not significantly different from the baseline value, both in patients who experienced and were studied at an exacerbation or in the overall population (Table 3; Fig. 1c, d). These results demonstrate that a natural exacerbation was associated with a modest increase in P-selectin that persisted for at least 6 weeks but later returned to baseline level. For a given individual, the mean $\mathrm{CV}$ for P-selectin among the 5 visits within these subjects was $27 \%$ and among the 3 nonexacerbation visits was $22 \%$, that is, the variation among visits in a subject was less than the variation among subjects at baseline.

\section{Discussion}

We found that high plasma-soluble P-selectin concentration at baseline (BPS) was associated with low $\mathrm{FEV}_{1}$ and with RV/TLC, an indicator of air trapping, in patients with asthma. These associations remained after adjustment for age and disease severity. Unlike FENO and sputum eosinophils, which are positively associated with clinical response, that is, improvement in $\mathrm{FEV}_{1}$, to corticosteroid [36], BPS was negatively associated with $\mathrm{FEV}_{1}$ change after systemic corticosteroid injection. That is, high BPS was associated with lack of improvement in $\mathrm{FEV}_{1}$ in response to systemic corticosteroid, whereas low BPS was associated with significant $\mathrm{FEV}_{1}$ improvement in response to corticosteroid. Elevated serum chitinaselike protein, YKL-40, has also recently been associated with lack of responsiveness to a 4-week asthma treatment regimen of inhaled corticosteroid (ICS) plus long-acting $\beta$-agonist [42]. Importantly, lack of corticosteroid responsiveness has recently been shown to be associated with and predict $\mathrm{FEV}_{1}$ decline in a SARP III networkwide study (Denlinger et al., unpublished).

BPS was highest in patients assigned to phenotype cluster 5 (called "severe fixed-airflow asthma" [33]) and predicted assignment to this cluster. BPS was not associated with indicators of type 2 immunity. In addition, neutrophils were not significantly different in BPS-high patients compared with BPS-low ones.

P-selectin was modestly but significantly elevated above baseline during a natural exacerbation and for at least 6 weeks afterward but later returned to baseline. The median increase was about $15-30 \%$ over the baseline value. As a comparison, the mean temporary rise in plasma P-selectin after whole-lung antigen challenge, which causes platelet activation [17], ranged from about 20 to $60 \%$ at $2-6 \mathrm{~h}$ in 2 studies $[16,17]$. This mean increase was likely higher because it was at the defined same times after challenge in each patient compared with the more modest median increase recorded here within 5 days after a natural exacerbation, which presumably was registered at somewhat varying times after the actual time of exacerbation in different patients. Few other molecular blood biomarkers have been found to be altered during and after a natural asthma exacerbation. Plasma monocyte chemotactic protein-4 (chemokine [C-C] motif ligand 13 [CCL13]) level was higher in patients with an acute exacerbation than in those with stable asthma [43].

There are several limitations of the present study. First, we used an in-house assay of P-selectin, albeit with reagents, protocol, and standard provided by a leading commercial provider. The stated cutoff concentrations, therefore, should be considered specific for our assay. Second, there is uncertainty of how to apply the results to the totality of asthma patients. Because our subjects were $>80 \%$ white non-Hispanics, to generalize our find-
P-Selectin Is Inversely Associated with Corticosteroid Responsiveness in Asthma
Int Arch Allergy Immunol 2020;181:879-887 DOI: $10.1159 / 000509600$ 
ings, a larger and more diverse cohort should be examined. Furthermore, the ability to discern biomarkers that correlate significantly with features of asthma will depend upon the mix and diversity of asthma patients being studied. As an example, P-selectin correlated with area of low density on chest CT at TLC in $59 \mathrm{~W}$ isconsin patients in SARP II [16]. However, this correlation did not hold up in the current study of SARP III patients; BPS and low density area did not correlate $\left(r_{\mathrm{s}}=-0.02, p=0.90\right)$ in the 62 patients on whom CT scans were performed. The makeup of the Wisconsin SARP II and III cohorts was different. In the SARP II cohort, $33 \%$ of the patients had severe asthma and 26\% were in the pooled clusters (No. 3-5) [16], whereas in the current SARP III cohort, 66\% of the patients were classified as severe (Table 1) and $44 \%$ were assigned to clusters $3-5$ with $20 \%$ in cluster 5 alone (Fig. 2a).

In summary, we propose that $\mathrm{P}$-selectin is a stable biomarker of a non-type 2 immunity asthma phenotype or endotype, characterized by airway obstruction/low $\mathrm{FEV}_{1}$, air trapping, lack of corticosteroid responsiveness, and the "severe fixed-airflow" cluster. The reasons for persistently high P-selectin in this group of asthma patients are not known but likely include systemic activation of endothelial cells and platelets.

\section{Acknowledgement}

The authors thank Holly Eversoll, Maranda Hyde, and Lori Wollet for patient recruitment, screening, and assessments; Jami Hauer, Renee Szakaly, and Heather Floerke for preparing plasma samples; Gina Crisafi and Tina Palas for administrative help and for providing subject data; and the SARP III DCC for providing subject data.

\section{Statement of Ethics}

Informed written consent was obtained from each subject before participation. This study was approved by the University of Wisconsin-Madison Health Sciences Institutional Review Board (Protocol No. 2012-0571).

\section{Conflict of Interest Statement}

MWJ received fees for consulting from Guidepoint Global and funds for research from Hoffmann-La Roche, none of which is related to P-selectin. LCD received fees from AstraZeneca and Sanofi-Regeneron, none of which is related to P-selectin. NNJ has received honoraria from Teva and AstraZeneca, none of which is related to P-selectin. The other authors declare no conflicts of interest.

\section{Funding Sources}

This study was supported by the National Institutes of Health (Grant No. U10 HL109168 to N.N.J.) and Clinical and Translational Science Award (Grant No. Ul1 RR025011 to M.K. Drezner) and in part by funds provided from the William W. Busse and Judith H. Busse Endowed Professorship in Allergy and Asthma Research (to L.C.D.). The funding source had no involvement in the data interpretation, the writing of the report, or the decision to submit the manuscript for publication.

\section{Author Contributions}

M.W.J., N.N.J., L.C.D., D.F.M., and R.L.S. conceived and designed the study and interpreted data. B.M.G., K.T.B., M.C.F., H.M.S., C.M.S., and M.W.J. acquired data and performed data analysis. M.W.J. and K.E.L. performed statistical analysis. M.W.J. drafted the manuscript. All authors revised the manuscript critically for important intellectual content and gave approval of the version to be submitted.

\section{References}

1 Ray A, Raundhal M, Oriss TB, Ray P, Wenzel SE. Current concepts of severe asthma. J Clin Invest. 2016;126(7):2394-403.

2 Svenningsen S, Nair P. Asthma endotypes and an overview of targeted therapy for asthma. Front Med. 2017;4:158

3 Akar-Ghibril N, Casale T, Custovic A, Phipatanakul W. Allergic endotypes and phenotypes of asthma. J Allergy Clin Immunol Pract. 2020;8(2):429-40.

4 Fitzpatrick AM, Chipps BE, Holguin F, Woodruff PG. T2-"low" asthma: overview and management strategies. J Allergy Clin Immunol Pract. 2020;8(2):452-63.

5 Fitzpatrick AM, Moore WC. Severe asthma phenotypes: how should they guide evaluation and treatment? J Allergy Clin Immunol Pract. 2017;5(4):901-8.
6 Chupp GL, Kaur R, Mainardi A. New therapies for emerging endotypes of asthma. Annu Rev Med. 2020;71:289-302.

7 Narendra D, Blixt J, Hanania NA. Immunological biomarkers in severe asthma. Semin Immunol. 2019;46:101332.

8 Diamant Z, Vijverberg S, Alving K, Bakirtas A, Bjermer L, Custovic A, et al. Toward clinically applicable biomarkers for asthma: an EAACI position paper. Allergy. 2019;74(10): 1835-51.

9 Silkoff PE, Moore WC, Sterk PJ. Three major efforts to phenotype asthma: Severe Asthma Research Program, Asthma Disease Endotyping for Personalized Therapeutics, and Unbiased Biomarkers for the Prediction of Respiratory Disease Outcome. Clin Chest Med. 2019;40(1):13-28.
10 Erjefält JS. Unravelling the complexity of tissue inflammation in uncontrolled and severe asthma. Curr Opin Pulm Med. 2019;25(1): 79-86.

11 Carr TF, Kraft M. Use of biomarkers to identify phenotypes and endotypes of severe asthma. Ann Allergy Asthma Immunol. 2018; 121(4):414-20.

12 Fahy JV. Type 2 inflammation in asthma present in most, absent in many. Nat Rev Immunol. 2015;15(1):57-65.

13 Kuo CS, Pavlidis S, Loza M, Baribaud F, Rowe A, Pandis I, et al. T-helper cell type 2 (Th2) and non-Th2 molecular phenotypes of asthma using sputum transcriptomics in U-BIOPRED. Eur Respir J. 2017;49(2): 1602135 . 
14 Kappelmayer J, Nagy B Jr, Miszti-Blasius K, Hevessy Z, Setiadi $H$. The emerging value of $\mathrm{P}$-selectin as a disease marker. Clin Chem Lab Med. 2004;42(5):475-86.

15 Johansson MW, Han ST, Gunderson KA, Busse WW, Jarjour NN, Mosher DF. Platelet activation, P-selectin, and eosinophil $\beta 1$ integrin activation in asthma. Am J Respir Crit Care Med. 2012;185(5):498-507.

16 Johansson MW, Kruger SJ, Schiebler ML, Evans MD, Sorkness RL, Denlinger LC, et al. Markers of vascular perturbation correlate with airway structural change in asthma. Am J Respir Crit Care Med. 2013;188(2):167-78.

17 Kowal K, Pampuch A, Kowal-Bielecka O, DuBuske LM, Bodzenta-Łukaszyk A. Platelet activation in allergic asthma patients during allergen challenge with Dermatophagoides pteronyssinus. Clin Exp Allergy. 2006;36(4): $426-32$.

18 Zietkowski Z, Skiepko R, Tomasiak MM, Bodzenta-Lukaszyk A. Soluble CD40 ligand and soluble P-selectin in allergic asthma patients during exercise-induced bronchoconstriction. J Investig Allergol Clin Immunol. 2008;18(4):272-8.

19 Sjöswärd KN, Uppugunduri S, Schmekel B. Decreased serum levels of $\mathrm{P}$-selectin and eosinophil cationic protein in patients with mild asthma after inhaled salbutamol. Respiration. 2004;71(3):241-5.

20 Mitsui C, Kajiwara K, Hayashi H, Ito J, Mita $\mathrm{H}$, Ono E, et al. Platelet activation markers overexpressed specifically in patients with aspirin-exacerbated respiratory disease. J Allergy Clin Immunol. 2016;137(2):400-11.

21 Palikhe S, Palikhe NS, Kim SH, Yoo HS, Shin YS, Park HS. Elevated platelet activation in patients with chronic urticaria: a comparison between aspirin-intolerant and aspirin-tolerant groups. Ann Allergy Asthma Immunol. 2014;113(3):276-81.

22 Andre P. P-selectin in haemostasis. Br J Haematol. 2004;126(3):298-306.

23 McEver RP, Beckstead JH, Moore KL, Marshall-Carlson L, Bainton DF. GMP-140, a platelet alpha-granule membrane protein, is also synthesized by vascular endothelial cells and is localized in Weibel-Palade bodies. J Clin Invest. 1989;84(1):92-9.
24 Hippenstiel S, Krüll M, Ikemann A, Risau W, Clauss M, Suttorp N. VEGF induces hyperpermeability by a direct action on endothelial cells. Am J Physiol. 1998;274(5):L678-84.

25 Kneuer C, Ehrhardt C, Radomski MW, Bakowsky U. Selectins: potential pharmacological targets? Drug Discov Today. 2006;11(2122):1034-40

26 Kanaji S, Fahs SA, Shi Q, Haberichter SL, Montgomery RR. Contribution of platelet vs. endothelial VWF to platelet adhesion and hemostasis. J Thromb Haemost. 2012;10(8): 1646-52.

27 Gearing AJ, Newman W. Circulating adhesion molecules in disease. Immunol Today. 1993;14(10):506-12.

28 Blann AD, Nitu-Whalley IC, Lee CA, Lip GY Inverse relationship between plasma von Willebrand factor and soluble $\mathrm{P}$ selectin in patients with type 1 but not type 2 von Willebrand disease. Am J Hematol. 2002;69(2): 135-7.

29 Kamath S, Blann AD, Caine GJ, Gurney D, Chin BS, Lip GY. Platelet P-selectin levels in relation to plasma soluble P-selectin and betathromboglobulin levels in atrial fibrillation. Stroke. 2002;33(5):1237-42.

30 Aleva FE, Temba G, de Mast Q, Simons SO, de Groot PG, Heijdra YF, et al. Increased platelet-monocyte interaction in stable COPD in the absence of platelet hyper-reactivity. Respiration. 2018;95(1):35-43.

31 Funderburg NT. Markers of coagulation and inflammation often remain elevated in ARTtreated HIV-infected patients. Curr Opin HIV AIDS. 2014;9(1):80-6.

32 McGroder CF, Aaron CP, Bielinski SJ, Kawut SM, Tracy RP, Raghu G, et al. Circulating adhesion molecules and subclinical interstitial lung disease: the Multi-Ethnic Study of Atherosclerosis. Eur Respir J. 2019;54(3): 1900295.

33 Moore WC, Meyers DA, Wenzel SE, Teague WG, Li H, Li X, et al. Identification of asthma phenotypes using cluster analysis in the Severe Asthma Research Program. Am J Respir Crit Care Med. 2010;181(4):315-23.

34 Gauvreau GM, Evans MY. Allergen inhalation challenge: a human model of asthma exacerbation. Contrib Microbiol. 2007;14:2132.
35 Teague WG, Phillips BR, Fahy JV, Wenzel SE, Fitzpatrick AM, Moore WC, et al. Baseline features of the Severe Asthma Research Program (SARP III) cohort: differences with age. J Allergy Clin Immunol Pract. 2018;6(2):54554.e4.

36 Phipatanakul W, Mauger DT, Sorkness RL, Gaffin JM, Holguin F, Woodruff PG, et al. Effects of age and disease severity on systemic corticosteroid responses in asthma. Am J Respir Crit Care Med. 2017;195(11):1439-48.

37 Denlinger LC, Phillips BR, Ramratnam S, Ross K, Bhakta NR, Cardet JC, et al. Inflammatory and comorbid features of patients with severe asthma and frequent exacerbations. Am J Respir Crit Care Med. 2017; 195(3):302-13.

38 Hastie AT, Mauger DT, Denlinger LC, Coverstone A, Castro M, Erzurum S, et al. Baseline sputum eosinophil + neutrophil subgroups' clinical characteristics and longitudinal trajectories for NHLBI Severe Asthma Research Program (SARP 3) cohort. J Allergy Clin Immunol. 2020;146(1):222-6.

39 Peters MC, McGrath KW, Hawkins GA, Hastie AT, Levy BD, Israel E, et al. Plasma interleukin-6 concentrations, metabolic dysfunction, and asthma severity: a cross-sectional analysis of two cohorts. Lancet Respir Med. 2016;4(7):574-84.

40 Sorkness RL, Bleecker ER, Busse WW, Calhoun WJ, Castro M, Chung KF, et al. Lung function in adults with stable but severe asthma: air trapping and incomplete reversal of obstruction with bronchodilation. J Appl Physiol. 2008;104(2):394-403.

41 Johansson MW, Evans MD, Crisafi GM, Holweg CTJ, Matthews JG, Jarjour NN. Serum periostin is associated with type 2 immunity in severe asthma. J Allergy Clin Immunol. 2016;137(6):1904-7.e2.

42 Liu L, Zhang X, Liu Y, Zhang L, Zheng J, Wang J, et al. Chitinase-like protein YKL-40 correlates with inflammatory phenotypes, anti-asthma responsiveness and future exacerbations. Respir Res. 2019;20(1):95.

43 Kalayci O, Sonna LA, Woodruff PG, Camargo CA Jr, Luster AD, Lilly CM. Monocyte chemotactic protein-4 (MCP-4; CCL-13): a biomarker of asthma. J Asthma. 2004;41(1):2733.
P-Selectin Is Inversely Associated with Corticosteroid Responsiveness in Asthma
Int Arch Allergy Immunol 2020;181:879-887 DOI: $10.1159 / 000509600$ 